\title{
On the Moduli Space of Elliptic Maxwell-Chern-Simons Theories
}

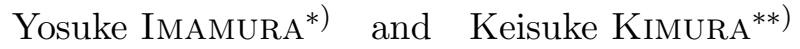 \\ Department of Physics, The University of Tokyo, Tokyo 113-0033, Japan
}

\begin{abstract}
We analyze the moduli space of the low-energy limit of 3-dimensional $\mathcal{N}=3$ MaxwellChern-Simons theories described by circular quiver diagrams, as for 4-dimensional elliptic models. We define the theories by using D3-NS5-(k,1)5-brane systems with an arbitrary number of fivebranes. The supersymmetry is expected to be enhanced to $\mathcal{N}=4$ in the low-energy limit. We show that the Higgs branch, in which all bifundamental scalar fields develop vacuum expectation values, is an abelian orbifold of $\mathbb{C}^{4}$. We confirm that the same geometry is obtained as an M-theory dual of the brane system. We also consider theories realized by introducing more than two kinds of fivebranes, and obtain nontoric fourfolds as moduli spaces.
\end{abstract}

\section{$\S 1$. Introduction}

Recently, there has been great interest in 3-dimensional superconformal field theories as theories for describing multiple M2-branes in various backgrounds. This was triggered by the proposal of a new class of 3-dimensional theories by Bagger and Lambert, ${ }^{1)-3)}$ and Gusstavson. ${ }^{4), 5)}$ The model (BLG model) possesses $\mathcal{N}_{(d=3)}=8$ superconformal symmetry and is based on Lie 3-algebra. The action of the BLG model includes the structure constant $f^{a b c}{ }_{d}$ of a Lie 3 -algebra, which determines the form of the interactions, and a metric $h^{a b}$, which appears in the coefficients of the kinetic terms. These tensors must satisfy certain conditions required by the supersymmetry invariance of the action. If these tensors satisfy the conditions, we can write down the action of a BLG model. The constraint imposed on the structure constant is called a fundamental identity. It was soon realized that the identity is very restrictive, ${ }^{6)}$ and it was proved that if we assume that the metric is positive definite and the algebra is finite dimensional, there is only one nontrivial Lie 3 -algebra, ${ }^{7}{ }^{, 8)}$ which is called an $A_{4}$ algebra. The BLG model based on the $A_{4}$ algebra is a $S U(2) \times S U(2)$ Chern-Simons theory with levels $k$ and $-k$ for each $S U(2)$ factor. Analysis of this model showed that it describes a pair of M2-branes in certain orbifold backgrounds. ${ }^{9)-11)}$ As a theory for an arbitrary number of M2-branes, a model based on an algebra with a Lorenzian metric was proposed in Refs. 12)14). Because of the indefinite metric, the model includes unwanted ghost modes. Although the ghost modes can be removed by treating them as background fields satisfying classical equations of motion, $\left.{ }^{14)}, 15\right)$ or by gauging certain symmetries and fixing them, $\left.{ }^{16)}, 17\right)$ this procedure breaks the conformal invariance, and the theory becomes D2-brane theory $\left.\left.{ }^{14)}, 16\right), 18\right)$ by the mechanism proposed in Ref. 19) unless the parameter corresponding to the Yang-Mills coupling is sent to infinity or integrated over all values as a dynamical parameter. ${ }^{17}$ )

\footnotetext{
*) E-mail: imamura@hep-th.phys.s.u-tokyo.ac.jp

**) E-mail: kimura@hep-th.phys.s.u-tokyo.ac.jp
} 
There has also been some progress in 3-dimensional Chern-Simons theories with supersymmetries of less than 8, which are closely related to M2-branes. Gaiotto and Witten ${ }^{20)}$ proposed $\mathcal{N}_{(\mathrm{d}=3)}=4$ superconformal Chern-Simons theories, and Hosomichi et al. ${ }^{21)}$ extended the theories by introducing twisted hypermultiplets. They derived the relation between their models and the BLG model, and showed that the $A_{4}$ BLG model is included as a special case of their $\mathcal{N}_{(d=3)}=4$ ChernSimons theories. They also studied the M-crystal model, ${ }^{22)-24)}$ which is described by a circular quiver diagram with $2 n$ vertices. The vertices represent Chern-Simons fields at level $\pm k$ with alternate signatures, and by analyzing the moduli space of the model they showed that it can be regarded as a theory describing M2-branes in the orbifold $\left(\mathbb{C}^{2} / \mathbb{Z}_{n}\right)^{2} *$ They also presented a realization of this model by using D3-, D5-, and NS5-branes, which give the model at level \pm 1 , and reproduce the orbifold as the M-theory dual of the brane system.

Aharony et al. also proposed a similar model ${ }^{25)}$ based on $U(N) \times U(N)$ ChernSimons theory with levels $k$ and $-k$ for each $U(N)$ factor. They showed that the action possesses $\mathcal{N}_{(d=3)}=6$ superconformal symmetry, and describes $N$ M2-branes in the orbifold $\mathbb{C}^{4} / \mathbb{Z}_{k}$. Although $\mathcal{N}_{(d=3)}=8$ supersymmetry, which is expected when $k=1$ or 2 is not manifest, the action does not have dimensionful parameters and the scale invariance is manifest. In Ref. 25) it is also shown that the theory can be realized as a theory on a brane system in type IIB string theory. The brane system consists of N D3-, one NS5-, and one $(k, 1) 5$-branes. They showed that by T-duality and M-theory lift, M2-branes in the orbifold $\mathbb{C}^{4} / \mathbb{Z}_{k}$ are obtained.

The purpose of this paper is to extend the models proposed in Refs. 21) and $25)$ by generalizing the brane configurations in these references. In 92 we consider a brane system with $n_{A}$ NS5-branes and $n_{B}(k, 1) 5$-branes, and analyze the moduli space of the theory realized by the brane system. The theory is a $U(N)^{n_{A}+n_{B}}$ quiver gauge theory with nonvanishing Chern-Simons terms for some of the $U(N)$ factors. Some of the $U(N)$ fields are Yang-Mills fields without Chern-Simons coupling. The supersymmetry of this theory is $\mathcal{N}_{(d=3)}=3$, which is expected to be enhanced to $\mathcal{N}_{(d=3)}=4$ in the strong gauge-coupling limit. The reason for this is as follows. This theory can be obtained from the $U(N) \times U(N)$ theory proposed in Ref. 20) by combining two extensions. One is the inclusion of twisted hypermultiplets, as mentioned above, and the other is the inclusion of gauge groups with vanishing Chern-Simons couplings. The latter extension is discussed in Ref. 20) to describe general nonlinear sigma models of hypermultiplets. Both extensions are known to give $\mathcal{N}_{(d=3)}=4$ supersymmetric Chern-Simons theory, and it is plausible that the theory we discuss in this paper possesses $\mathcal{N}_{(d=3)}=4$ supersymmetry.

In 93 we determine the moduli space of the theory. We focus only on the Higgs branch, which describes a mobile M2-brane. Under a certain assumption for flux quantization, we obtain a 4 -dimensional orbifold $\mathbb{C}^{4} / \Gamma$, where $\Gamma$ is a discrete subgroup depending on $k, n_{A}$, and $n_{B}$. We reproduce the same orbifold in $₫ 4$ as an M-theory dual of the brane configuration. In $\$ 5$ we consider models with more than

*) The possibility that the existence of magnetic monopoles causes a discrete indentification in the orbifold is also mentioned. 


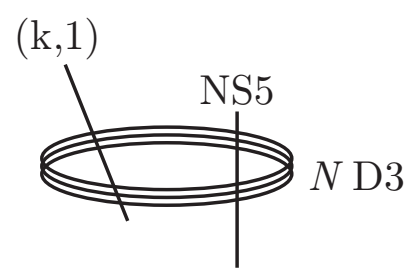

Fig. 1. Brane configuration for the $U(N) \times U(N)$ Chern-Simons model.

two kinds of fivebranes. The moduli space is also a 4-dimensional manifold, but it is nontoric. The last section is devoted to discussion.

\section{$\S 2$. Brane configuration and action}

The model proposed in Ref. 25) is a Chern-Simons theory with a $U(N) \times U(N)$ gauge group. It can be realized as a theory based on a brane system consisting of $N$ D3-branes, one NS5-brane, and one $(k, 1) 5$-brane. All these branes share the directions of 012 , which are the coordinates of the 3-dimensional field theory. The $N$ D3-branes are wrapped on the compact direction 9 . The NS5-brane and the $(k, 1) 5$ brane are spread along the 012345 and $012[36]_{\theta_{1}}[47]_{\theta_{2}}[58]_{\theta_{3}}$ directions, respectively, where $[i j]_{\theta}$ is the direction in the $i-j$ plane specified by the angle $\theta$. The angles $\theta_{1,2,3}$ are determined by the BPS conditions. We refer to NS5- and $(k, 1) 5$-branes as A- and B-branes, respectively. The D3-brane worldvolume is divided into two parts by the intersecting fivebranes (Fig. (1), and a $U(N)$ vector multiplet exists on each segment. Bifundamental chiral multiplets also arise at the intersections. This brane system is similar to the D4-NS5 system realizing the Klebanov-Witten theory, ${ }^{26)}$ which is a 4-dimensional $\mathcal{N}_{(d=4)}=1$ superconformal field theory. In the D4-NS5 system, we have $N$ D4-branes wrapped on $\mathbb{S}^{1}$, instead of D3-branes, and the A- and B-branes in this case are NS5-branes along different directions.

We generalize the D3-fivebrane system by introducing an arbitrary number of fivebranes. In the case of 4 -dimensional $\mathcal{N}_{(d=4)}=1$ gauge theories, such a generaliza-

tion is known as an elliptic model, and has been studied in detail. ${ }^{27), 28)}$ It is known that the moduli spaces of the theories are generalized conifolds. We here carry out a similar analysis in the 3 -dimensional case. Let $n_{A}$ and $n_{B}$ be the numbers of A- and B-branes, respectively. We denote the total number of fivebranes by $n=n_{A}+n_{B}$. Let us label the fivebranes by $I=1, \ldots, n$ according to their order along $\mathbb{S}^{1}$. We identify $I=n+1$ with $I=1$. On the interval of D3-branes between two fivebranes $I$ and $I+1$, we have a $U(N)$ vector multiplet $V_{I}$ and an adjoint chiral multiplet $\Phi_{I}$. (We use the terminology of $\mathcal{N}_{(d=4)}=(1 / 2) \mathcal{N}_{(d=3)}=1$ supersymmetry.) The kinetic terms of these multiplets are

$$
\begin{aligned}
& S_{V}=\int d^{3} x \sum_{I} \frac{1}{g_{I}^{2}} \operatorname{tr}\left[-\frac{1}{4}\left(F_{\mu \nu}^{I}\right)^{2}-\frac{1}{2}\left(D_{\mu} \sigma_{I}\right)^{2}+\frac{1}{2} D_{I}^{2}+\text { fermions }\right] \\
& S_{\Phi}=\int d^{3} x d^{4} \theta \sum_{I} \frac{1}{g_{I}^{2}} \operatorname{tr}\left(\Phi_{I}^{*} e^{V_{I}} \Phi_{I} e^{-V_{I}}\right) .
\end{aligned}
$$




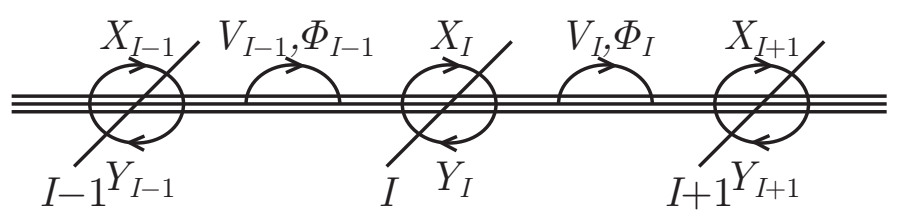

Fig. 2. Brane system and fields.

$\sigma_{I}$ is the real scalar field in the vector multiplet $V_{I}$. The adjoint chiral multiplets $\Phi_{I}$ describe the motion of the D-branes along the fivebranes. When two fivebranes $I$ and $I+1$ are not parallel, the chiral multiplet $\Phi_{I}$ becomes massive, and the mass term is described by the superpotential

$$
W=\frac{\mu}{2} \sum_{I}\left(q_{I+1}-q_{I}\right) \Phi_{I}^{2},
$$

where $q_{I}=0$ for A-branes and $q_{I}=1$ for B-branes. The overall factor $\mu$ is related to the relative angle between A- and B-branes.

We also have bifundamental chiral multiplets $X_{I}$ and $Y_{I}$, which arise from open strings stretched between two intervals of D-branes divided by the $I$ th fivebrane. (See Fig. 2.) These fields belong to the following representations of $U(N)_{I} \times U(N)_{I-1}$, where $U(N)_{I}$ is the gauge group associated with the vector multiplet $V_{I}$ :

$$
X_{I}:(N, \bar{N}), \quad Y_{I}:(\bar{N}, N) .
$$

The kinetic terms of these bifundamental fields are

$$
\begin{aligned}
S_{X Y}= & \int d^{3} x d^{4} \theta \sum_{I=1}^{n} \operatorname{tr}\left[X_{I}^{*} e_{I}^{V} X_{I} e^{-V_{I-1}}+Y_{I} e^{-V_{I}} Y_{I}^{*} e^{V_{I-1}}\right] \\
= & \int d^{3} x \sum_{I=1}^{n} \operatorname{tr}\left[-D_{I}\left(\left|X_{I}\right|^{2}-\left|Y_{I}\right|^{2}-\left|X_{I+1}\right|^{2}+\left|Y_{I+1}\right|^{2}\right)\right. \\
& \left.-\left(\left|X_{I}\right|^{2}+\left|Y_{I}\right|^{2}\right)\left(\sigma_{I}-\sigma_{I-1}\right)^{2}+\left|F_{I}^{X}\right|^{2}+\left|F_{I}^{Y}\right|^{2}\right]+\cdots .
\end{aligned}
$$

In the component expression we show only the bosonic terms without derivatives. These bifundamental fields couple to the adjoint chiral multiplets through the superpotential

$$
W=\sum_{I=1}^{n} \operatorname{tr} \Phi_{I}\left(X_{I} Y_{I}-Y_{I+1} X_{I+1}\right)
$$

The difference between the RR-charges of the A- and B-branes generates ChernSimons terms. ${ }^{29), 30)}$ The bosonic part of the $\mathcal{N}_{d=3}=2$ completion of the ChernSimons terms is

$$
S_{\mathrm{CS}}=\sum_{I=1}^{n} \frac{k_{I}}{2 \pi} \int d^{3} x \operatorname{tr}\left[\epsilon^{\mu \nu \rho}\left(\frac{1}{2} A_{\mu}^{I} \partial_{\nu} A_{\rho}^{I}+\frac{1}{3} A_{\mu}^{I} A_{\nu}^{I} A_{\rho}^{I}\right)+\sigma_{I} D_{I}\right],
$$

where the Chern-Simons coupling $k_{I}$ is given by

$$
k_{I}=k\left(q_{I+1}-q_{I}\right) \text {. }
$$


We assume that $k$ is a positive integer. The Chern-Simons terms in (2·7) cause some of the vector multiplets to be massive. The masses $\sim k_{I} g_{I}^{2}$ are proportional to the masses of adjoint chiral multiplets $\Phi_{I}$. We can promote the supersymmetry of this theory to $\mathcal{N}_{(d=3)}=3$ by matching the masses of $V_{I}$ and $\Phi_{I}$ by setting $\mu=k$.

In 3-dimensional field theories the coupling constants $g_{I}$ have mass dimension $1 / 2$, and taking the low-energy limit is equivalent to taking the strong-coupling limit $g_{I} \rightarrow \infty$. This makes the masses of $V_{I}$ and $\Phi_{I}$ infinity unless $k_{I}=0$, and we can integrate out the massive adjoint chiral multiplets. After this, the superpotential becomes*)

$$
W=\sum_{q_{I}=q_{I+1}} \operatorname{tr} \Phi_{I}\left(X_{I} Y_{I}-Y_{I+1} X_{I+1}\right)+\sum_{q_{I} \neq q_{I+1}}\left(q_{I+1}-q_{I}\right) \operatorname{tr}\left(X_{I} Y_{I} Y_{I+1} X_{I+1}\right) .
$$

\section{$\S 3 . \quad$ Moduli space}

In this section we investigate the moduli space of the 3-dimensional field theory defined in the previous section. As we mentioned at the end of the previous section we need to take the strong-coupling limit $g_{I} \rightarrow \infty$ to obtain the conformal theory describing the low-energy limit of M2-branes. Although the dynamics in such a strong coupling region is highly nontrivial, we assume that the vacuum structure is not affected by quantum corrections, and we consider only the classical equations of motion derived from the action given in the previous section. In the strong-coupling limit, the kinetic terms $(2 \cdot 1)$ and $(2 \cdot 2)$ vanish, and the fields $\phi_{I}$, the scalar components of $\Phi_{I}$, and $\sigma_{I}$ become auxiliary fields. The bifundamental chiral multiplets $X_{I}$ and $Y_{I}$ are still dynamical, and the moduli space is parameterized by the scalar components of these multiplets.

We are interested in the moduli space for a single M2-brane, and we set $N=1$. Furthermore, we here focus only on the Higgs branch, which describes a mobile M2-brane, and assume

$$
X_{I}, Y_{I} \neq 0
$$

\subsection{F-term conditions}

Let us first consider the F-term conditions derived from the superpotential (2.9). Because the superpotential is the same as the 4-dimensional elliptic model realized by the D4-NS5 brane system, the F-term conditions are also the same. Under the assumption (3.1), the F-term conditions give the following solution:

$$
\Phi_{I \in A}=M_{I \in B}=u, \quad \Phi_{I \in B}=M_{I \in A}=v,
$$

where we define the mesonic operators as $M_{I}=X_{I} Y_{I} . I \in A(I \in B)$ means that index $I$ is restricted to the values with $q_{I}=0\left(q_{I}=1\right)$.

Although not directly related to our model, it may be instructive to demonstrate how we can obtain a Calabi-Yau 3-fold as the moduli space of a 4-dimensional elliptic model in the case of the D4-NS5 system. In this case two complex numbers $u$ and $v$

*) We shift the field $\Phi_{I}$ by $\left(q_{I}-1 / 2\right)\left(X_{I} Y_{I}+Y_{I+1} X_{I+1}\right)$ and set $\mu=1$ to simplify the equations. 


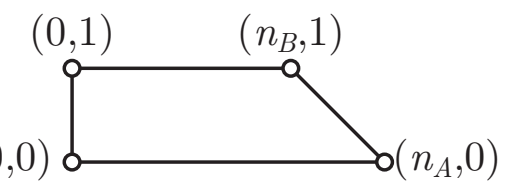

Fig. 3. Toric diagram of a generalized conifold.

can be interpreted as the coordinates of the D4-brane along B- and A-branes, respectively. The 4-dimensional theory possesses $U(1)^{n-1}$ gauge symmetry. In addition to the mesonic operators $M_{I}$, we can construct the gauge-invariant baryonic operators

$$
x=\prod_{I=1}^{n} X_{I}, \quad y=\prod_{I=1}^{n} Y_{I} .
$$

By definition, these gauge-invariant operators are related by

$$
x y=u^{n_{A}} v^{n_{B}} .
$$

This algebraic equation defines a Calabi-Yau 3-fold, which is often called a generalized conifold. The toric diagram of this generalized conifold is shown in Fig. 3 ,

\subsection{D-term conditions}

In the strong-coupling limit $g_{I} \rightarrow \infty$, the vector multiplet $V_{I}$ includes two auxiliary fields $\sigma_{I}$ and $D_{I}$. The terms in the action including these auxiliary fields are

$$
\begin{aligned}
S= & \sum_{I=1}^{n}\left[k_{I} \sigma_{I} D_{I}-D_{I}\left(\left|X_{I}\right|^{2}-\left|Y_{I}\right|^{2}-\left|X_{I+1}\right|^{2}+\left|Y_{I+1}\right|^{2}\right)\right. \\
& \left.-\left(\left|X_{I}\right|^{2}+\left|Y_{I}\right|^{2}\right)\left(\sigma_{I}-\sigma_{I-1}\right)^{2}\right] .
\end{aligned}
$$

In this action, $D_{I}$ are Lagrange multipliers, and give the constraint

$$
k_{I} \sigma_{I}=\left|X_{I}\right|^{2}-\left|Y_{I}\right|^{2}-\left|X_{I+1}\right|^{2}+\left|Y_{I+1}\right|^{2} .
$$

If we substitute this into the action $(\underline{3 \cdot 5})$, the first line vanishes and the potential becomes

$$
V=\sum_{I=1}^{n}\left(\left|X_{I}\right|^{2}+\left|Y_{I}\right|^{2}\right)\left(\sigma_{I}-\sigma_{I-1}\right)^{2} .
$$

Because of the assumption (3.1), vacua are given by $\sigma_{I}=\sigma_{I-1}$. Namely, all $\sigma_{I}$ are the same. Let $\sigma$ be the common value of $\sigma_{I}$. Then the constraint $(\underline{3 \cdot 6})$ becomes

$$
q_{I} \sigma-\left(\left|X_{I}\right|^{2}-\left|Y_{I}\right|^{2}\right)=q_{I+1} \sigma-\left(\left|X_{I+1}\right|^{2}-\left|Y_{I+1}\right|^{2}\right) .
$$

This means that the left- and right-hand sides of this equation do not depend on the index $I$. Thus, we can write

$$
\left|X_{I}\right|^{2}-\left|Y_{I}\right|^{2}=q_{I} \sigma+c
$$


with a constant $c$.

Although (3.9) is not the equation of motion of $D_{I}$, we can formally interpret it as an ordinary D-term condition associated with a certain symmetry. To rewrite $(3 \cdot 9)$ in the form of an ordinary D-term condition, let us define $U(1)$ transformation groups $G_{I}$ that act only on $X_{I}$ and $Y_{I}$ as

$$
G_{I}: X_{I} \rightarrow e^{i \lambda_{I}} X_{I}, \quad Y_{I} \rightarrow e^{-i \lambda_{I}} Y_{I},
$$

where $\lambda_{I}$ is a parameter of $G_{I}$. The groups $G_{I}$ are different from $U(1)_{I}$ defined in the previous section. The parameters $\alpha_{I}$ of $U(1)_{I}$ and $\lambda_{I}$ of $G_{I}$ are related by

$$
\lambda_{I}=\alpha_{I}-\alpha_{I-1} .
$$

Although each $G_{I}$ is not a symmetry of the theory, it is convenient to describe symmetry groups as subgroups of $\prod_{I} G_{I}$. For example, the gauge symmetry $G=$ $U(1)^{n-1}$, which does not include the diagonal $U(1)$ decoupling from the theory, is the subgroup of $\prod_{I} G_{I}$ that does not rotate the baryonic operators (3.3).

Let us rewrite $(\underline{3 \cdot 9})$ in the form of a D-term condition. Equation $(\underline{3.9})$ is equivalent to the condition

$$
\sum_{I=1}^{l} \lambda_{I}\left(\left|X_{I}\right|^{2}-\left|Y_{I}\right|^{2}\right)=0
$$

for arbitrary $\lambda_{I}$ satisfying the constraints

$$
\sum_{I=1}^{n} \lambda_{I}=\sum_{I=1}^{n} q_{I} \lambda_{I}=0 .
$$

If we regard $\lambda_{I}$ as the parameters of $G_{I}$ transformations, the constraints (3.13) imposed on $\lambda_{I}$ define a subgroup $H=U(1)^{n-2}$ of $\prod_{I} G_{I}$. Equation (3.12) can be regarded as the D-term condition for $H$.

We emphasize that we do not claim at this point that the gauge symmetry of the theory is $H$ or that relation (3.9) is obtained as the equations of motion of auxiliary fields in the vector multiplets associated with $H$. We only claim that the vacuum condition (3.9) is similar to the D-term condition of a gauge theory with the gauge symmetry $H$. In the next subsection, however, we will show that $H$ indeed emerges as the unbroken continuous gauge symmetry.

It is convenient to define the subgroup $H$ in another way. Let us define the baryonic operators

$$
x_{A}=\prod_{I \in A} X_{I}, \quad y_{A}=\prod_{I \in A} Y_{I}, \quad x_{B}=\prod_{I \in B} X_{I}, \quad y_{B}=\prod_{I \in B} Y_{I} .
$$

The group $H$ can be defined as the subgroup of $\prod_{I} G_{I}$ that does not rotate these baryonic operators.

\subsection{Gauge symmetry}

To obtain the moduli space of a gauge theory, we need to remove unphysical degrees of freedom corresponding to gauge symmetries. In the case of Chern-Simons 
theories, we should carefully take account of symmetry breaking due to the existence of magnetic monopoles. Let us rewrite the abelian Chern-Simons terms in the form

$$
S_{\mathrm{CS}}=-\frac{k}{2 \pi} \sum_{I=1}^{n} q_{I}\left(A^{I}-A^{I-1}\right) \wedge \widetilde{F}+\left(\text { quadratic terms of } A^{I}-A^{I-1}\right),
$$

where $\widetilde{F}$ is the field strength of the diagonal $U(1)$ gauge field $\widetilde{A}=(1 / n)\left(A^{1}+A^{2}+\right.$ $\left.\cdots+A^{n}\right)$. Equation (3.15) is obtained by substituting

$$
A^{I}=\widetilde{A}+\left(\text { linear combination of } A^{I}-A^{I-1}\right)
$$

into the Chern-Simons term in (2.7). The quadratic term of $\widetilde{A}$ vanishes because $\sum_{I} k_{I}=0$. Because the diagonal gauge field $\widetilde{A}$ appears only in the first term of $(\underline{3 \cdot 15})$, we can dualize it by adding the term

$$
\frac{1}{2 \pi} \int d \tau \wedge \widetilde{F}
$$

and treating $\widetilde{F}$ as an unconstrained field. The equation of motion of $\widetilde{F}$ gives

$$
\sum_{I=1}^{n} k_{I} A_{I}=d \tau
$$

Upon the gauge transformation $\delta A_{I}=d \alpha_{I}$, the scalar field $\tau$ is transformed as

$$
\delta \tau=\sum_{I=1}^{n} k_{I} \alpha_{I}
$$

Let us assume that the period of $\tau$ is $2 \pi$. This implies that the flux $\oint \widetilde{F}$ is quantized by

$$
\int \widetilde{F} \in 2 \pi \mathbb{Z}
$$

Although we could not show this flux quantization on the field-theory side, we will later show that the moduli space obtained by assuming (3.20) coincides with that obtained from the brane configuration by the T-duality and M-theory lift. If we adopt this assumption, the gauge fixing $\tau=0$ partially breaks the gauge symmetry and imposes the following constraint on the parameters $\lambda_{I}$ and $\alpha_{I}$ :

$$
\sum_{I=1}^{n} k_{I} \alpha_{I}=k \sum_{I=1}^{n} q_{I} \lambda_{I} \in 2 \pi \mathbb{Z}
$$

(In the first equality we used (2.8) and (3.11).)

Let us first focus on the continuous subgroup. It is generated by parameters satisfying

$$
\sum_{I=1}^{n} \lambda_{I}=\sum_{I=1}^{n} q_{I} \lambda_{I}=0
$$


The group defined by $(3 \cdot 22)$ is simply group $H$ defined in 3.2 . Because of the emergence of the same group $H$ both in the equations of motion of auxiliary fields and in the unbroken gauge symmetry, we can obtain the moduli space as the coset $\mathcal{M} / H_{\mathbb{C}}$ or its orbifold, where $\mathcal{M}$ is the complex manifold defined by the F-term conditions and $H_{\mathbb{C}}$ is the complexification of the group $H$. This guarantees that the moduli space is a complex manifold.

In addition to $H$, the group defined by (3.21) includes the discrete symmetry, which rotates the baryonic operators in (3.14) as

$$
x_{A} \rightarrow e^{\frac{2 \pi i}{k}} x_{A}, \quad y_{A} \rightarrow e^{-\frac{2 \pi i}{k}} y_{A}, \quad x_{B} \rightarrow e^{-\frac{2 \pi i}{k}} x_{B}, \quad y_{B} \rightarrow e^{\frac{2 \pi i}{k}} y_{B} .
$$

\subsection{Moduli space}

Let us determine the moduli space. We first consider the $k=1$ case. In this case, the discrete gauge symmetry (3.23) becomes trivial, and we have the gauge-invariant operators

$$
u, \quad v, \quad x_{A}, \quad y_{A}, \quad x_{B}, y_{B} .
$$

By definition, these operators satisfy the following equations:

$$
x_{A} y_{A}=u^{n_{A}}, \quad x_{B} y_{B}=v^{n_{B}} .
$$

These equations define the orbifold $\mathbb{C}^{2} / \mathbb{Z}_{n_{A}} \times \mathbb{C}^{2} / \mathbb{Z}_{n_{B}}$. Actually, the relation (3.25) can be solved as

$$
x_{A}=z_{1}^{n_{A}}, \quad y_{A}=z_{2}^{n_{A}}, \quad u=z_{1} z_{2}, \quad x_{B}=z_{3}^{n_{B}}, \quad y_{B}=z_{4}^{n_{B}}, \quad v=z_{3} z_{4} .
$$

We can identify $z_{i}$ as the coordinates of $\mathbb{C}^{4}$, the covering space of the orbifold. None of the variables in (3.24) are changed by the transformations

$$
\left(z_{1}, z_{2}, z_{3}, z_{4}\right) \rightarrow\left(e^{2 \pi i / n_{A}} z_{1}, e^{-2 \pi i / n_{A}} z_{2}, z_{3}, z_{4}\right)
$$

and

$$
\left(z_{1}, z_{2}, z_{3}, z_{4}\right) \rightarrow\left(z_{1}, z_{2}, e^{2 \pi i / n_{B}} z_{3}, e^{-2 \pi i / n_{B}} z_{4}\right) .
$$

Points in $\mathbb{C}^{4}$ mapped by these transformations should be identified with each other, and this identification defines the above orbifold.

If $n_{A}=n_{B}$, the moduli space agrees with the result in Ref. 21), in which alternate $\mathrm{A}$ - and B-branes are considered. It is interesting that the moduli space does not depend on the order of the two kinds of fivebranes.

Next, let us consider the case when $k>1$. In this case, we should take account of the discrete gauge transformation (3.23). The transformation of $z_{i}$ reproducing $(3 \cdot 23)$ is

$$
\left(z_{1}, z_{2}, z_{3}, z_{4}\right) \rightarrow\left(e^{2 \pi i / k n_{A}} z_{1}, e^{-2 \pi i / k n_{A}} z_{2}, e^{-2 \pi i / k n_{B}} z_{3}, e^{2 \pi i / k n_{B}} z_{4}\right) .
$$

The three transformations (3.27), (3.28), and (3.29) generate a discrete subgroup of $U(1)^{2}$ with $k n_{A} n_{B}$ elements. Let $\Gamma$ be this discrete group. The moduli space for general $k$ is the abelian orbifold $\mathbb{C}^{4} / \Gamma$. 


\section{$\S 4 . \quad$ M-theory dual}

In the previous section, we obtained the 4 -dimensional orbifold $\mathbb{C}^{4} / \Gamma$ as the Higgs branch of the moduli space. The purpose of this section is to reproduce the same orbifold by the T-duality transformation and the M-theory lift from the D3fivebrane system in type IIB string theory.

For simplicity, we first consider a system in which the $(k, 1) 5$-branes are replaced by D5-branes. After determining the mapping from type IIB string theory to Mtheory for NS5- and D5-branes, the dual object for the bound state of these two kinds of branes is easily obtained by superposing the objects for NS5- and D5-branes. Although the tilted angle of the branes should be appropriately chosen according to the charges of branes to preserve supersymmetry, we do not do this because the toric data do not change upon continuous deformations of the manifold and because we can determine the toric data of the dual geometry by using only the topological information. We start from the brane configuration for type IIB string theory in Table I. Direction 9 is compactified on $\mathbb{S}^{1}$. We replaced the $(k, 1) 5$-brane with the D5-brane and use a coordinate system in which the D5-brane is spread along 012678. In general, the $(k, 1) 5$-branes are not perpendicular to the NS5-brane, thus we use slanted coordinates.

We first rearrange the coordinates in 4578 space by using the Hopf fibration. We define $r_{a}(a=1,2,3)$ by

$$
r_{a}=u^{\dagger} \sigma_{a} u, \quad u=\left(\begin{array}{c}
x^{4}+i x^{5} \\
x^{7}+i x^{8}
\end{array}\right)
$$

and we let $\psi$ be the coordinate of the $\mathbb{S}^{1}$ fiber. Then the NS5 and D5 worldvolumes are on the positive and negative parts of the $r_{3}$ axis, respectively, in the $r_{a}$ space. See Table II. The $\psi$ cycle shrinks at the center of the $r_{a}$ space, which is shown in the table as "KKM". "s" in the table represents the shrinking cycle.

Let us perform the T-duality transformation along direction 9, and lift the configuration into M-theory. The D3-branes are mapped to M2-branes as shown in

Table I. Brane configuration in type IIB string theory

\begin{tabular}{c|ccc|ccc|ccc|c} 
& 0 & 1 & 2 & 3 & 4 & 5 & 6 & 7 & 8 & 9 \\
\hline D3 & $\circ$ & $\circ$ & $\circ$ & & & & & & & $\circ$ \\
D5 & $\circ$ & $\circ$ & $\circ$ & & & & $\circ$ & $\circ$ & $\circ$ & \\
NS5 & $\circ$ & $\circ$ & $\circ$ & $\circ$ & $\circ$ & $\circ$ & & & &
\end{tabular}

Table II. The same configuration as Table 1 with a different coordinate system. "s" represents the shrinking cycle and + and - mean that the branes are spread along the positive or negative part of the axis, respectively.

\begin{tabular}{c|ccc|ccc|cc|cc} 
& 0 & 1 & 2 & 3 & 6 & $r_{3}$ & $r_{1}$ & $r_{2}$ & $\psi$ & 9 \\
\hline D3 & $\circ$ & $\circ$ & $\circ$ & & & & & & & $\circ$ \\
D5 & $\circ$ & $\circ$ & $\circ$ & & $\circ$ & - & & & $\circ$ & \\
NS5 & $\circ$ & $\circ$ & $\circ$ & $\circ$ & & + & & & $\circ$ & \\
KKM & $\circ$ & $\circ$ & $\circ$ & $\circ$ & $\circ$ & & & & $\mathrm{s}$ & $\circ$
\end{tabular}


On the Moduli Space of Elliptic Maxwell-Chern-Simons Theories

Table III. M-theory dual of the brane configuration.

\begin{tabular}{c|ccc|ccc|cc|ccc} 
& 0 & 1 & 2 & 3 & 6 & $r_{3}$ & $r_{1}$ & $r_{2}$ & $\psi$ & 9 & $\mathrm{M}$ \\
\hline$(\mathrm{D} 3 \rightarrow) \mathrm{M} 2$ & $\circ$ & $\circ$ & $\circ$ & & & & & & & & \\
$(\mathrm{D} 5 \rightarrow) \mathrm{KKM}$ & $\circ$ & $\circ$ & $\circ$ & & $\circ$ & - & & & $\circ$ & $\mathrm{s}$ & $\circ$ \\
$(\mathrm{NS} 5 \rightarrow) \mathrm{KKM}$ & $\circ$ & $\circ$ & $\circ$ & $\circ$ & & + & & & $\circ$ & $\circ$ & $\mathrm{s}$ \\
$\mathrm{KKM}$ & $\circ$ & $\circ$ & $\circ$ & $\circ$ & $\circ$ & & & & $\mathrm{s}$ & $\circ$ & $\circ$
\end{tabular}

Table III. A single NS5-brane and a single D5-brane become KKM-branes associated with the $(1,0,0)$ and $(0,1,0)$ cycles, respectively, where the first, second, and last components correspond to the $M, 9$, and $\psi$ coordinates, respectively. If we start with a $(k, 1) 5$-brane, which is the bound state of $k$ D5-branes and one NS5-brane, we obtain a single KKM-brane with $(0,1, k)$ cycle shrinking.

In addition to these, we have one more KKM-brane, which originates from the special choice of the coordinates. The existence of the other KKM-branes make the shrinking cycle of the last KKM-brane ambiguous, and only the last component of the shrinking cycle has a definite value of 1 . The intersection with other branes changes the shrinking cycle, and the cycle should be determined according to the "charge conservation" of the KKM branes. See Fig. 4(a).

This system of KKM-branes in $36 r_{3}$ space is simply a webdiagram describing a 4-dimensional toric manifold. We can easily obtain the toric diagram as a dual graph of the webdiagram. (Fig. 4(b)) The toric variety described by this diagram is in fact the orbifold we obtained in 93.4 , as we show in the rest of this section.

The structure of a toric variety is mostly determined by the toric data, which are a set of generators of shrinking cycles. The generators are usually represented as vectors $\vec{v}_{i}$ in the lattice associated with the toric fiber. The toric data of $\mathbb{C}^{4}$ are given by $\vec{v}_{i}=\vec{e}_{i}(i=1,2,3,4)$, where $\vec{e}_{i}$ are the unit vectors in the 4 -dimensional lattice.

$$
\vec{e}_{1}=(1,0,0,0), \quad \vec{e}_{2}=(0,1,0,0), \quad \vec{e}_{3}=(0,0,1,0), \quad \vec{e}_{4}=(0,0,0,1)
$$

The orbifolding of a toric variety is realized by refining the lattice by adding new generators. In the case of the orbifold defined by (3.27)-(3.29), we add three gener-

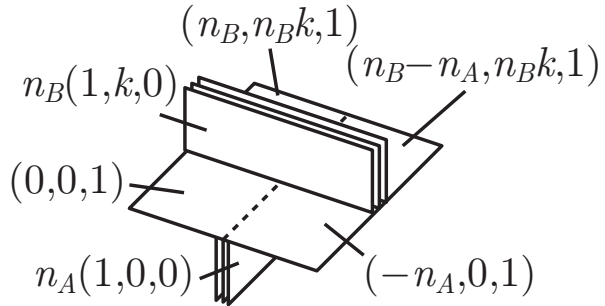

(a)

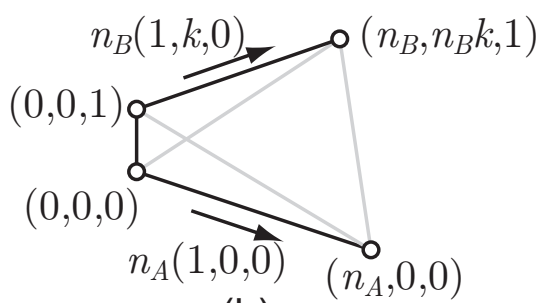

(b)

Fig. 4. (a) M-theory dual of the D3-fivebrane system in $36 r_{3}$ space. This can be regarded as a webdiagram of the toric geometry. The corresponding toric diagram is shown in (b). 
ators

$$
\begin{aligned}
& \vec{e}_{5}=\left(\frac{1}{n_{A}},-\frac{1}{n_{A}}, 0,0\right), \\
& \vec{e}_{6}=\left(0,0, \frac{1}{n_{B}},-\frac{1}{n_{B}}\right), \\
& \vec{e}_{7}=\left(\frac{1}{n_{A} k},-\frac{1}{n_{A} k},-\frac{1}{n_{B} k}, \frac{1}{n_{B} k}\right) .
\end{aligned}
$$

Of course, the seven vectors $\vec{e}_{1}, \ldots, \vec{e}_{7}$ are not linearly independent. Let us choose the following linearly independent basis:

$$
\begin{aligned}
& \overrightarrow{f_{1}}=-\vec{e}_{5}=\left(-\frac{1}{n_{A}}, \frac{1}{n_{A}}, 0,0\right), \\
& \overrightarrow{f_{2}}=\vec{e}_{7}=\left(\frac{1}{n_{A} k},-\frac{1}{n_{A} k},-\frac{1}{n_{B} k}, \frac{1}{n_{B} k}\right), \\
& \overrightarrow{f_{3}}=\vec{e}_{3}-\vec{e}_{1}=(-1,0,1,0), \\
& \overrightarrow{f_{4}}=\vec{e}_{1}=(1,0,0,0) .
\end{aligned}
$$

Using this basis, the toric data become

$$
\vec{v}_{1}=[0,0,0,1]_{\vec{f}}, \quad \vec{v}_{2}=\left[n_{A}, 0,0,1\right]_{\vec{f}}, \quad \vec{v}_{3}=[0,0,1,1]_{\vec{f}}, \quad \vec{v}_{4}=\left[n_{B}, k n_{B}, 1,1\right]_{\vec{f}},
$$

where $\left[a_{1}, \cdots, a_{4}\right]_{\vec{f}}=\sum_{i} a_{i} \vec{f}_{i}$. We have chosen basis (4.4) so that the toric data become the standard form in which the last components of the vectors are 1 . We can draw the toric diagram using the first three components of these vectors $\vec{v}_{i}$, which coincides with that in Fig. 4(b).

\section{$\S 5$. Further generalization}

Up to now we have considered a brane system with two kinds of fivebranes. It is also possible to introduce more than two kinds of fivebranes. To represent the types of branes we used $q_{I}=0$ and 1 . In this section we allow $q_{I}$ to be an arbitrary integer. In this case, we do not need to introduce the coefficient $k$ in (2.8) and we set $k=1$. This means that the $I$ th fivebrane is a $\left(q_{I}, 1\right) 5$-brane, and the Chern-Simons couplings are given by

$$
k_{I}=q_{I+1}-q_{I} .
$$

For simplicity we assume that the Chern-Simons couplings do not vanish. This implies that all the adjoint chiral multiplets $\Phi_{I}$ and the vector multiplets $V_{I}$ become massive. It is easy to show that even if some of the $k_{I}$ vanish we obtain the same moduli space as derived below.

By integrating out $\Phi_{I}$, we obtain the superpotential

$$
W=-\sum_{I=1}^{n} \frac{1}{2\left(q_{I+1}-q_{I}\right)}\left(X_{I} Y_{I}-Y_{I+1} X_{I+1}\right)^{2} .
$$


(When we obtained the superpotential (2.9) we used $q_{I}=0$ and 1 , although we cannot use it here.) From the assumption (3.1), the F-term conditions for $X_{I}$ and $Y_{I}$ give

$$
\frac{X_{I+1} Y_{I+1}-X_{I} Y_{I}}{q_{I+1}-q_{I}}=\frac{X_{I} Y_{I}-X_{I-1} Y_{I-1}}{q_{I}-q_{I-1}},
$$

and this is solved as

$$
X_{I} Y_{I}=a+q_{I} b
$$

where $a$ and $b$ are arbitrary complex numbers.

The equations of motion of the auxiliary fields $\sigma_{I}$ are solved by $(3 \cdot 12)$ with the parameters $\lambda_{I}$ constrained by (3.13). The constraint (3.13) defines group $H$ and Eq. (3.12) has the form of the D-term condition associated with group $H$. This group is identical to the continuous part of the unbroken gauge symmetry, which is given by (3.21) with $k=1$. The constraints imposed on the parameters are

$$
\sum_{I=1}^{n} \lambda_{I}=0, \quad \sum_{I=1}^{n} q_{I} \lambda_{I} \in \frac{1}{2 \pi} \mathbb{Z} .
$$

The following "baryonic operators" are invariant under gauge symmetries satisfying (5.5),

$$
\begin{array}{ll}
x=\prod_{I=1}^{n} X_{I}, \quad x_{A}=\prod_{I=1}^{n} X_{I}^{q_{\max }-q_{I}}, \quad x_{B}=\prod_{I=1}^{n} X_{I}^{q_{I}-q_{\min }}, \\
y=\prod_{I=1}^{n} Y_{I}, \quad y_{A}=\prod_{I=1}^{n} Y_{I}^{q_{\max }-q_{I}}, \quad y_{B}=\prod_{I=1}^{n} Y_{I}^{q_{I}-q_{\min }},
\end{array}
$$

where $q_{\min }$ and $q_{\max }$ are the minimum and maximum of $q_{I}$, respectively. Any gaugeinvariant monomial of $X_{I}$ and $Y_{I}$ can be represented as a monomial of the mesonic operators $M_{I}=X_{I} Y_{I}$ and these baryonic operators.

We now have the following 8 gauge-invariant variables:

$$
a, b, x, x_{A}, x_{B}, y, y_{A}, y_{B}
$$

These operators satisfy

$$
\begin{gathered}
x y=\prod_{I=1}^{n}\left(a+q_{I} b\right), \quad x_{A} y_{A}=\prod_{I=1}^{n}\left(a+q_{I} b\right)^{q_{\max }-q_{I}}, \quad x_{B} y_{B}=\prod_{I=1}^{n}\left(a+q_{I} b\right)^{q_{I}-q_{\min }}, \\
x_{A} x_{B}=x^{q_{\max }-q_{\min }}, \quad y_{A} y_{B}=y^{q_{\max }-q_{\min }} .
\end{gathered}
$$

Because the first equation in (5.9) is not independent of the other two due to relations (5.10), these relations decrease the number of independent degrees of freedom by four, and the moduli space becomes a complex 4-dimensional space. If the $q_{I}$ take more than two different values, the equations in (5.9) are not binary relations of monomials, and the moduli space is nontoric. 


\section{$\S 6 . \quad$ Discussion}

In this paper we studied the Higgs branch of Maxwell-Chern-Simons theories described by circular quiver diagrams. We first considered the model realized by the D3-NS5- $(k, 1) 5$-brane system with an arbitrary number of fivebranes, and showed that the moduli space is the orbifold $\mathbb{C}^{4} / \Gamma$, where $\Gamma$ is the discrete group generated by (3.27)-(3.29). When we determined the orbifold group $\Gamma$, we made an assumption for the flux quantization (3.20). Our result was confirmed by comparing it to the M-theory dual of the brane configuration. We also discussed the model realized by a brane system with more than two kinds of fivebranes, and we obtained a 4dimensional nontoric moduli space.

Note that our result is different from that expected from the orbifold method. In general, a quiver gauge theory obtained by the orbifold method introduced in Ref. 31) includes $n$ copies of fields of the parent theory, where $n$ is the order of the orbifolding group. Such analysis is carried out in Ref. 32) for the model proposed in Ref. 25), and a theory was obtained in which the number of $U(N)$ factors in the gauge group is proportional to the order of the corresponding orbifolding group. On the other hand, our construction gives $n=n_{A}+n_{B}$ copies of fields, whereas the order of the orbifolding group is proportional to the product $n_{A} n_{B}$. Because the brane construction and orbifold method are both important methods for constructing field theories in string theory, it is very important to understand the reason for this discrepancy.

The moduli spaces we obtained in this paper are completely determined by the number of fivebranes of each type. In the case of the brane system with A- and Bbranes, the moduli space depends only on the level $k$ and the numbers of fivebranes $n_{A}$ and $n_{B}$. The orders of A- and B-branes along the compact direction do not affect the moduli space. This is also the case in the brane system with more than two types of fivebranes discussed in 95 . This may be interpreted as a duality similar to the Seiberg duality in the 4 -dimensional $\mathcal{N}_{(d=4)}=1$ supersymmetric gauge theories. ${ }^{33)}$ In the 4-dimensional case, this duality can be understood as the exchange of the two types of branes. ${ }^{34)}$ In the brane system we consider in this paper, the exchange of A- and B-branes generates new D3-branes by the Hanany-Witten effect. ${ }^{35)}$ It will be an interesting problem to clarify the relation among theories realized by brane systems with different orders of fivebranes.

The models we considered in this paper are expected to flow to conformal fixed points in the low-energy limit, and thus the AdS/CFT correspondence is expected to be useful for studying low-energy dynamics. When we discuss the AdS/CFT correspondence, it is necessary to establish the correspondence between geometries and the UV description of quiver gauge theories. In the case of 4-dimensional $\mathcal{N}=$ 1 superconformal theories, brane tiling ${ }^{36)}{ }^{38)}$ is a convenient tool for finding this correspondence in the toric Calabi-Yau case. Although the generalization of brane tiling to 3-dimensional gauge theories has been proposed, ${ }^{22-24)}$ much less is known about the duality in the 4-dimensional case due to the small number of examples. We hope that the examples in this paper will be useful for investigating the general relation between four-manifolds and quiver Chern-Simons gauge theories. 
On the Moduli Space of Elliptic Maxwell-Chern-Simons Theories

\section{Acknowledgements}

We would like to thank T. Eguchi for valuable discussions. We would also like to acknowledge the helpful comments of K. Ohta. Y. I. is partially supported by a Grant-in-Aid for Young Scientists (B) (\#19740122) from the Japan Ministry of Education, Culture, Sports, Science and Technology.

\section{References}

1) J. Bagger and N. Lambert, Phys. Rev. D 75 (2007), 045020; hep-th/0611108.

2) J. Bagger and N. Lambert, Phys. Rev. D 77 (2008), 065008; arXiv:0711.0955

3) J. Bagger and N. Lambert, J. High Energy Phys. 02 (2008), 105; arXiv:0712.3738

4) A. Gustavsson, arXiv:0709.1260.

5) A. Gustavsson, J. High Energy Phys. 04 (2008), 083; arXiv:0802.3456

6) P. M. Ho, R. C. Hou and Y. Matsuo, arXiv:0804.2110

7) G. Papadopoulos, J. High Energy Phys. 05 (2008), 054; arXiv:0804.2662

8) J. P. Gauntlett and J. B. Gutowski, arXiv:0804.3078.

9) M. Van Raamsdonk, J. High Energy Phys. 05 (2008), 105; arXiv:0803.3803

10) N. Lambert and D. Tong, arXiv:0804.1114.

11) J. Distler, S. Mukhi, C. Papageorgakis and M. Van Raamsdonk, J. High Energy Phys. 05 (2008), 038; arXiv:0804.1256.

12) J. Gomis, G. Milanesi and J. G. Russo, arXiv:0805.1012

13) S. Benvenuti, D. Rodriguez-Gomez, E. Tonni and H. Verlinde, arXiv:0805.1087

14) P. M. Ho, Y. Imamura and Y. Matsuo, arXiv:0805.1202.

15) Y. Honma, S. Iso, Y. Sumitomo and S. Zhang, arXiv:0805.1895.

16) M. A. Bandres, A. E. Lipstein and J. H. Schwarz, arXiv:0806.0054.

17) J. Gomis, D. Rodriguez-Gomez, M. Van Raamsdonk and H. Verlinde, arXiv:0806.0738

18) B. Ezhuthachan, S. Mukhi and C. Papageorgakis, arXiv:0806.1639.

19) S. Mukhi and C. Papageorgakis, J. High Energy Phys. 05 (2008), 085; arXiv:0803.3218.

20) D. Gaiotto and E. Witten, arXiv:0804.2907.

21) K. Hosomichi, K. M. Lee, S. Lee, S. Lee and J. Park, arXiv:0805.3662.

22) S. Lee, Phys. Rev. D 75 (2007), 101901; hep-th/0610204.

23) S. Lee, S. Lee and J. Park, J. High Energy Phys. 05 (2007), 004; hep-th/0702120.

24) S. Kim, S. Lee, S. Lee and J. Park, Nucl. Phys. B 797 (2008), 340; arXiv:0705.3540.

25) O. Aharony, O. Bergman, D. L. Jafferis and J. Maldacena, arXiv:0806.1218

26) I. R. Klebanov and E. Witten, Nucl. Phys. B 536 (1998), 199; hep-th/9807080

27) A. M. Uranga, J. High Energy Phys. 01 (1999), 022; hep-th/9811004.

28) R. von Unge, J. High Energy Phys. 02 (1999), 023; hep-th/9901091.

29) T. Kitao, K. Ohta and N. Ohta, Nucl. Phys. B 539 (1999), 79; hep-th/9808111

30) O. Bergman, A. Hanany, A. Karch and B. Kol, J. High Energy Phys. 10 (1999), 036; hep-th/9908075

31) M. R. Douglas and G. W. Moore, hep-th/9603167

32) M. Benna, I. Klebanov, T. Klose and M. Smedback, arXiv:0806.1519.

33) N. Seiberg, Nucl. Phys. B 435 (1995), 129; hep-th/9411149

34) S. Elitzur, A. Giveon and D. Kutasov, Phys. Lett. B 400 (1997), 269; hep-th/9702014

35) A. Hanany and E. Witten, Nucl. Phys. B 492 (1997), 152; hep-th/9611230.

36) A. Hanany and K. D. Kennaway, hep-th/0503149

37) S. Franco, A. Hanany, K. D. Kennaway, D. Vegh and B. Wecht, J. High Energy Phys. 01 (2006), 096; hep-th/0504110

38) S. Franco, A. Hanany, D. Martelli, J. Sparks, D. Vegh and B. Wecht, J. High Energy Phys. 01 (2006), 128; hep-th/0505211. 